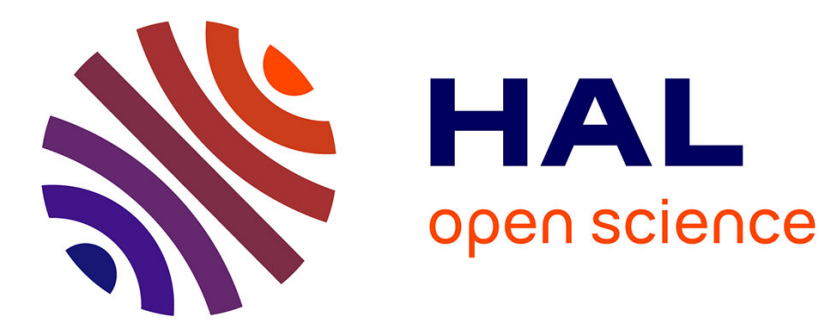

\title{
Towards the prediction of fluctuating wall quantities using Immersed Boundary Conditions
}

\author{
Lucas Manueco, Pierre-Elie Weiss, Sébastien Deck
}

\section{To cite this version:}

Lucas Manueco, Pierre-Elie Weiss, Sébastien Deck. Towards the prediction of fluctuating wall quantities using Immersed Boundary Conditions. AIAA AVIATION 2019, Jun 2019, DALLAS, United States. 10.2514/6.2019-3700 . hal-02333686

\section{HAL Id: hal-02333686 https://hal.science/hal-02333686}

Submitted on 25 Oct 2019

HAL is a multi-disciplinary open access archive for the deposit and dissemination of scientific research documents, whether they are published or not. The documents may come from teaching and research institutions in France or abroad, or from public or private research centers.
L'archive ouverte pluridisciplinaire HAL, est destinée au dépôt et à la diffusion de documents scientifiques de niveau recherche, publiés ou non, émanant des établissements d'enseignement et de recherche français ou étrangers, des laboratoires publics ou privés. 


\title{
Towards the prediction of fluctuating wall quantities using Immersed Boundary Conditions
}

\author{
Lucas Manueco*, Pierre-Élie Weiss ${ }^{\dagger}$ and Sébastien Deck ${ }^{*}$ \\ ONERA-The French Aerospace Lab, F-92190 Meudon, France
}

\begin{abstract}
Immersed boundary conditions (IBC) has reached a sufficient level of maturity to allow the simulation of compressible high Reynolds number flows around complex geometries. However, the reconstruction of physical quantities at the wall of geometries introduced using IBC is far from being straightforward. The difficulty to obtain a prediction as accurate as for classical body-fitted approaches originates from the intrinsic mismatch between immersed boundaries and the underlying mesh. To tackle this issue, a novel method to compute global loads and to provide precise wall data in the view of spectral analyses is introduced. First, this method is assessed for the investigation of highly unsteady separating compressible flows of two space launcher afterbody configurations using Zonal Detached Eddy Simulation (ZDES). Then, the results are compared against validated numerical simulations using a classical body-fitted approach. Finally, the present method successfully returns wall quantities with IBC consistent with classical methodologies and without additional time-consuming operations.
\end{abstract}

\section{Introduction}

The knowledge of fluctuating quantities at the wall is of first importance for the design of launch vehicles. As an example, space launcher afterbodies are subject to strong effects issuing from recirculation zones induced by sudden geometrical changes. The pressure fluctuations resulting from this phenomenon generates significant load variations which can affect the launcher stability. The location and the magnitude of this variation are highly dependent on the launcher shape and have to be assessed for each new launch vehicle. Classical body-fitted methods coupled with Zonal Detached Eddy Simulation (ZDES) which belongs to the hybrid RANS/LES (Reynolds Averaged Navier-Stokes/Large Eddy Simulation) approaches has been used successfully to quantify the dynamic load [1]. With this numerical strategy, Weiss and Deck [2] identified the main frequencies of this phenomenon and their location with single and two-point spectral analysis on a generic afterbody configuration corroborating the experiment of Meliga and Reijasse [3].

Nowadays, the development of new space launchers requires the computation and the analysis of several configurations in the context of Multidisciplinary Design Optimisation (MDO) in order to reduce the final number of experimental test cases. Nonetheless, the mesh generation for structured methodologies may be complex and time-consuming for complex geometries. Different approaches such as Immersed Boundary Conditions (IBC) can be used in order to limit the time devoted to the mesh generation process.

During the last decades, IBC became a standard method to extend the capacity of a CFD solver to handle complex geometries. The boundaries are imposed in the near wall region of non-body fitted grid. IBC applications can be found for compressible, high Reynolds number steady and unsteady flows. Mochel et al. [4] have shown the interest of a zonal application of IBC (ZIBC) in order to quantify the effect of a control device on an afterbody flow. More recently this approach was used by Weiss and Deck [5] on a pre-existing body-fitted simulation to add the technological details of a full space launcher geometry. IBC have been found to retrieve successfully mean aerodynamic values and second-order statistics whereas the reconstruction of integral forces and the spectral content on immersed boundaries remain poorly documented.

For body-fitted methods, the mesh provides the knowledge of both elementary surfaces and the normal vectors of the geometry in order to integrate the pressure and the shear stress tensor which are mandatory to predict forces. Due to the mismatch between the IBC and the mesh, the integration surface required for global load computations is not explicitly given by the underlying mesh. To reach the analysis level of body-fitted methods, it is needed to develop efficient methods to retrieve fluctuating wall values for immersed boundaries. The final objective is to get access to the global load and assess their capacity to obtain higher validation levels such as single and two-point spectral analyses.

\footnotetext{
*Ph.D. Student, Aerodynamics, Aeroelasticity, Acoustics Department, lucas.manueco@onera.fr

$\dagger$ Senior Research Scientist, Aerodynamics, Aeroelasticity, Acoustics Department, pierre-elie.weiss@onera.fr

${ }^{\ddagger}$ Senior Research Scientist, Aerodynamics, Aeroelasticity, Acoustics Department, sebastien.deck@onera.fr
} 
The present paper aims to introduce a methodology which allows performing wall analyses for unsteady simulations as it would be achieved with body-fitted approaches. This method is applied to simulate and analyse the unsteady phenomena generating pressure fluctuations on two space-launcher afterbodies using ZDES. The paper is organized as follows. Section 2 details the numerical treatment used to access to the fluctuating wall values and global loads. Section 3 describes the two configurations used as validation test cases. Section 4 is devoted to the numerical aspects of the simulations. Finally, section 5 exhibits the wall analysis of the two launcher afterbodies using IBC and the comparison against validated body-fitted simulations.

\section{Immersed Boundary Surfaces}

Forcing points (FP) are used to enforce the immersed boundary condition in the computational domain. FP flow field values are constraint by mean of source terms to enforce the wall condition without mesh body-fitting. FP locations can be found inside [6] or outside [7] the immersed solid and, in the particular case of finite volume methods, at the cell faces between solid and fluid cells [8, 9]. The constraint values are set using neighbouring fluid cells and an interpolation procedure is used in order to reconstruct the flow state in the wall-normal directions through FP as represented in figure 1. As in a classical body-fitted method, an assumption is made on the evolution of the variables in the wall normal direction to impose the value at the FP. In the literature, linear velocity variation, as well as wall-models, have been used for IBC [6, 8].

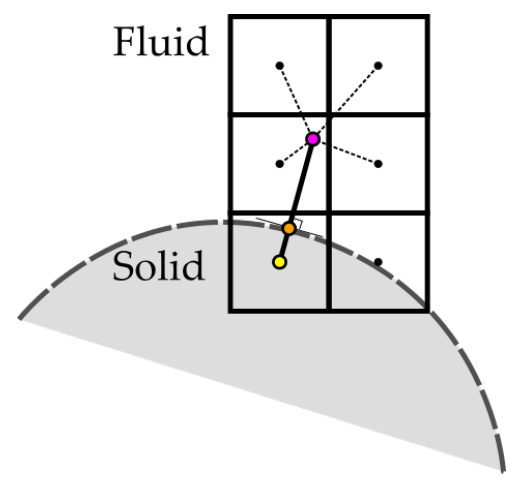

Fig. 1 Representation of the interpolation procedure for IBC. O: Interpolation Points (IntP). O: No-Slip Points (NSP). O: Forcing Points (FP) inside the solid.

The no-slip immersed boundary condition is imposed at the so-called No-Slip Points (NSP) represented in figure 1 , and are taken into account during the computation of the FP values. The values at the actual immersed wall are only known during the computation process and are not retrieved in the flow variables. Moreover, a difference has to be made between the initial geometry given to the flow solver and the geometry in the solver. Since wall conditions are imposed only in one point by FP, the representation of the initial geometry is modified by the IBC as presented in figure 2. Results using wall-model based IBC are particularly influenced by this discrepancy since the mesh requirements are lower than the one obtained with linear velocity variation assumptions. Therefore, both the IBC method and the mesh refinement have to be taken into account to produce an accurate representation of the real body surface.

As a first approach, an interpolation of the flow variables on the initial geometry can be used but do not respect the two previously discuss points. This approach is highly sensitive to the interpolation method and the near-wall mesh refinement. Another possibility which is particularly attractive with IBC lies in the use of indirect load computation since no information at the wall is necessary. This approach has been used successfully by Tamaki et al. [9] to compute the lift and drag coefficient on a wing profile. Nonetheless, the difference between viscous and inviscid effects and the local load contribution are not trivially provided by indirect methods. Several authors proposed to approximate the integration surface using the background mesh [10, 11]. More recently Capizzano [12] suggested to create a surface mesh linking the NSP in order to create a surface mesh of the immersed wall. Thus, this surface mesh can be used as in a body-fitted method to compute the required surface information for global load computation and to extract wall quantities.

During the computation of the source terms applied to the FP, the values at NSP are explicitly known using the same assumption that for the FP to provide wall values to the immersed surface mesh without interpolations. This method has 


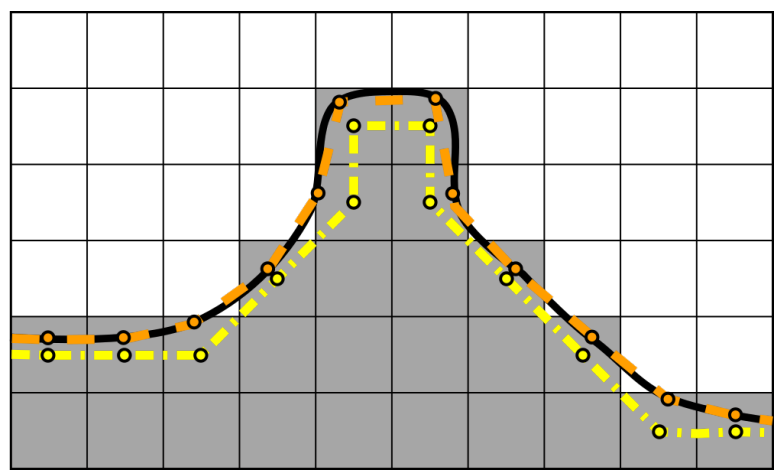

Fig. 2 Scheme of the influence of the discretization on the geometry representation. O: Forcing points (FP) inside the solid. O: No-slip points.

been developed for the particular case of Cartesian grids with FP located at the cell-faces between solid and fluid cells [12]. However, the accuracy of this method to compute global loads has not been assessed so far.

A new algorithm is here presented to adapt the method for inside and outside FP. In order to generate the mesh with connectivity between the NSP, a first grid is created between the FP (see Fig. 2) with algorithm 11 The main idea consists in linking FP sharing the same vertices. It is worth noting that a restriction test, presented in algorithm 1, has to be performed in order to avoid false connections. Figure 3 depicts an example of the limitation effect of the restriction test.

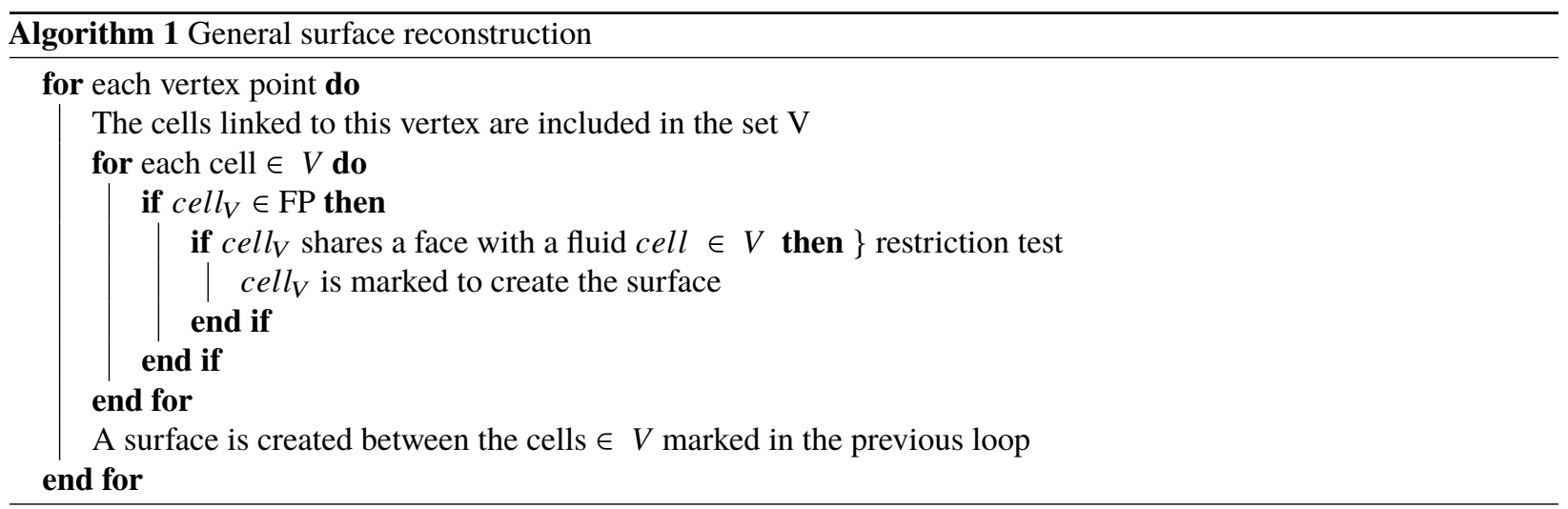

Algorithm 1 can be used for structured and unstructured grids in the particular case of inside FP. Algorithm 2 presents a modified restriction test for outside FP. As proposed by Capizzano [12], the connectivity table between FP can be transferred to their corresponding NSP in order to create the final immersed surface mesh. During the computation process, the NSP flow field values are computed and sent to the surface grid. As a result, classical body-fitted methods can be used to process the global load and wall quantity extraction without any additional cost during the computation compared to classical methodologies.

If multiple layers of FP are used in order to impose the IBC, only the first layer is considered to generate the surface. The surface mesh at the wall can be created for all the FP layers with simple modifications of the restriction test. However, the merging of the meshes can cause unbalanced surface distributions for the NSP. As a consequence, the accuracy of the global load reconstruction can be decreased. Thus, such a situation has to be avoided.

\section{Test cases}

To assess the capacity of the present approach to provide accurate analyses at the wall with IBC for unsteady computations, two configurations were considered. The first one, called Generic Afterbody (GA) configuration is designed to fit the experiment of Meliga et al. [3] conducted in ONERA's S3Ch wind tunnel. The configuration is composed of two cylinders of size $D$ equal to $100 \mathrm{~mm}$ and $0.4 D$. The smaller cylinder is an extension of the first 


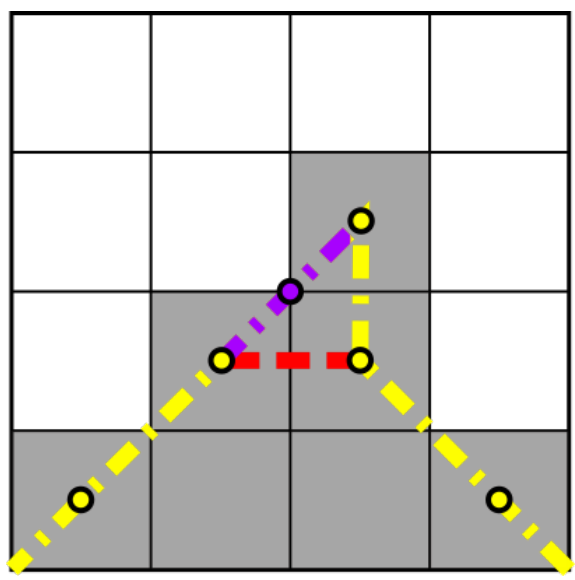

Fig. 3 Illustration of the restriction test of algorithm 1 O: Forcing points (FP) inside the solid. $\mathrm{O}$ : Vertex of interest. $=-$ : FP connections. $\cdots+\cdots$ : Erroneous connection. $\cdots+\cdots$ : Connection currently created.

cylinder of size $L / D=1.2$. The length of the bigger cylinder is chosen in order to obtain a boundary layer thickness ratio $\delta / D$ of 0.2 at the separation point. This axisymmetric case is simulated with a Mach number of 0.702 for the free stream flow and a corresponding diameter based Reynolds number $R e_{D}$ of $1.2 \cdot 10^{6}$. Such a configuration has been studied with a body-fitted approach by Weiss and Deck [2, 13]. Due to the sudden change of geometry at the end of the first cylinder, the smaller cylinder is subject to unsteady load variations. In the present simulation, the smaller cylinder is modeled using IBC whereas the bigger cylinder is modeled using a body-fitted method as represented in figure 4 The objective of this first case is to assess the accuracy of the reconstruction method in the most favourable case since the mesh is aligned with the IBC.

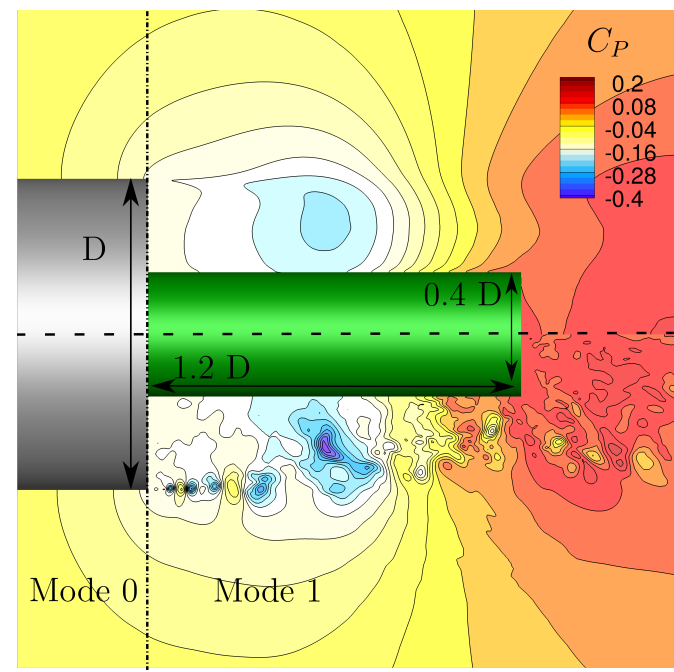

(a)

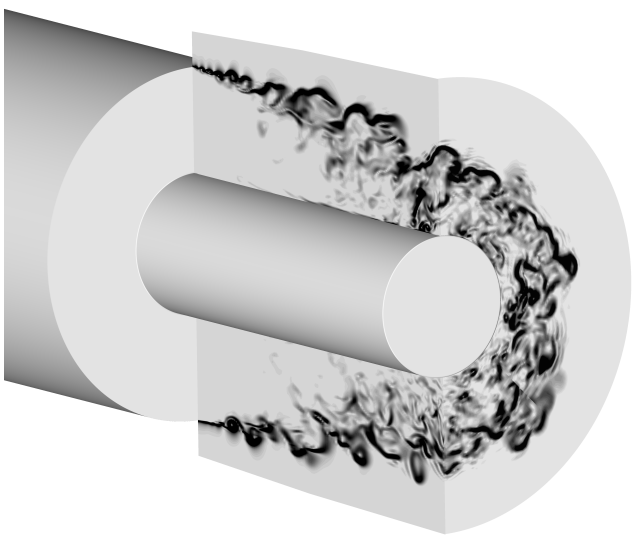

(b)

Fig. 4 (a) Contours of the pressure coefficient $C_{p}$ (upper part: mean flow field, lower part: instantaneous flow field) (b) numerical schlieren for the GA configuration (grey part: Body-fitted boundary, green part: Immersed Boundary)

Then, to evaluate the methodology on a more complex geometry, a Realistic Afterbody (RA) corresponding to a "smooth" Ariane 5 afterbody (namely without technological details) was chosen and replace the smaller cylinder of the GA configuration (see Fig. 5). The free stream Mach number is increased to 0.8 and the length of the bigger cylinder is truncated in order to match the numerical simulation of Weiss and Deck [14] with a $\delta / D$ ratio of 0.1. 


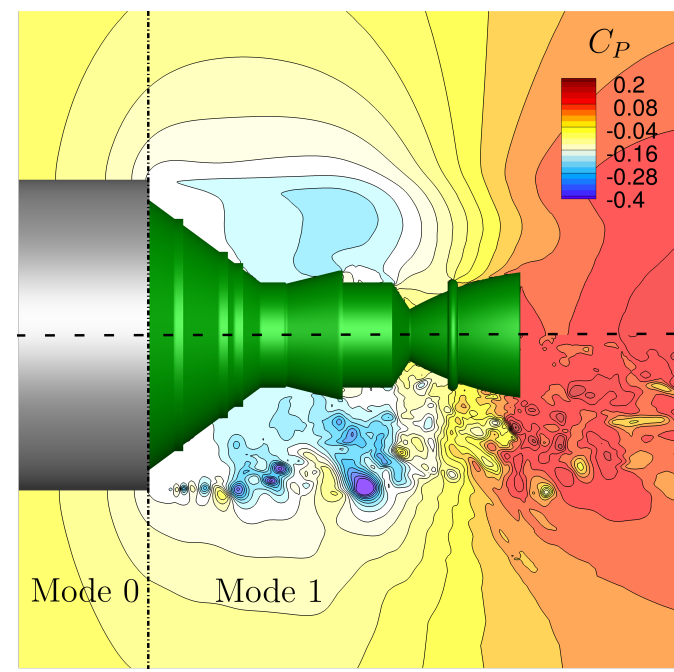

(a)

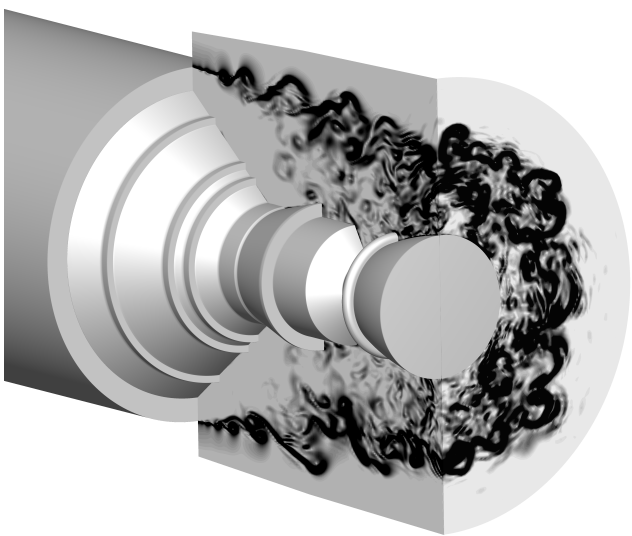

(b)

Fig. 5 (a) Contours of the pressure coefficient $C_{p}$ (upper part: mean flow field, lower part: instantaneous flow field) (b) numerical schlieren for the RA configuration (grey part: Body-fitted boundary, green part: Immersed Boundary)

\section{Numerical Method}

\section{A. CFD Code}

The FLU3M code used for the present simulations is a finite-volume solver for the compressible Navier-Stokes equations on structured grids. An AUSM(P)+ scheme proposed by Liou [15] coupled with a MUSCL reconstruction without limiter, is employed for the inviscid flux computation. The viscous fluxes are assessed using the second order Green-Gauss method. A second order implicit backward scheme Gear presented by Péchier et al. [16] is used for the time integration. The FLU3M code has been validated on various applications [17, 18] and more particularly for space launcher simulations [19, 20]. All numerical simulations are performed without changes between the resolution methods.

\section{B. Zonal Detached Eddy Simulation (ZDES)}

The approach used to model the flow under consideration is the Zonal Detached Eddy Simulation (ZDES) proposed by Deck [21, 22]. ZDES has been evaluated for the simulation of complex turbulent flows [23]. It has proved to simulate high Reynolds number configurations at an affordable cost [22]. Contrary to non-zonal hybrid RANS/LES methods, ZDES offers a capacity to switch between the different modeling techniques of the turbulence flow in the same simulation. The upstream part of the flow, corresponding to the turbulent boundary layer on the bigger cylinder is modeled using an URANS approach corresponding to ZDES mode 0. The massively separated flow around the afterbody is treated using LES for the study of the pressure fluctuation corresponding to mode 1 of the ZDES.

\section{Immersed Boundary Method}

A discrete forcing immersed boundary method has been selected and developed in the CFD solver FLU3M. This approach is derived from the work of Mohd-Yusof [24] and proposes to impose the boundary condition by mean of source terms. The present method uses inside forcing points without wall models. This approach has been successfully used to reproduce the blockage effect of control devices on a space launcher afterbody by Mochel et al. [25]. More recently, this approach was used by Weiss and Deck [5] on a pre-existing body-fitted simulation to add the geometrical details of a full space launcher geometry. This approach coupling a body-fitted simulation and IBC, called Zonal Immersed Boundary Condition (ZIBC), has shown to reproduce the mean and fluctuating quantities of the experimental configuration satisfactorily. Nonetheless, most of the launchers were simulated using body-fitted method whereas in the present article the area of interest is fully treated using IBC. 


\section{Results and Discussion}

\section{A. Instantaneous and averaged flow fields}

Figures 4 and 5 propose a qualitative overview of the instantaneous and time-averaged flow fields with iso-contours of the pressure coefficient on a longitudinal cut for both configurations. Kelvin-Helmholtz instabilities of the mixing layer appear in both cases at the end of the upstream cylinder. The streamlines of the time-averaged field exhibit the same salient features as for the body-fitted simulations namely recirculation zones and a reattachment point on the extension. Figures 6 and 7 show a comparison between streamwise mean pressure coefficient and the rms pressure coefficient. In both cases, the pressure coefficient is in good agreement with the body-fitted computation. For the GA case, the Root Mean Square (rms) pressure coefficient is slightly overestimated. The rise of rms values is related to the growth of shear layer structures in the near-wall region around the reattaching point at $x / D=1$ as explained by Weiss et al. [13]. The impact of those structures on the IBC seems to have a strong effect on the $C p_{r m s}$ distribution for $x / D>0.9$ as observed in figure 6 . The RA case reproduces qualitatively the streamwise evolution of the rms pressure coefficient with an overall underestimation of the values. The representation of the various sharp edges of the geometry is not as well-modeled as in the body-fitted computation which can explain the smaller peak at the various geometrical changes of the RA configuration.
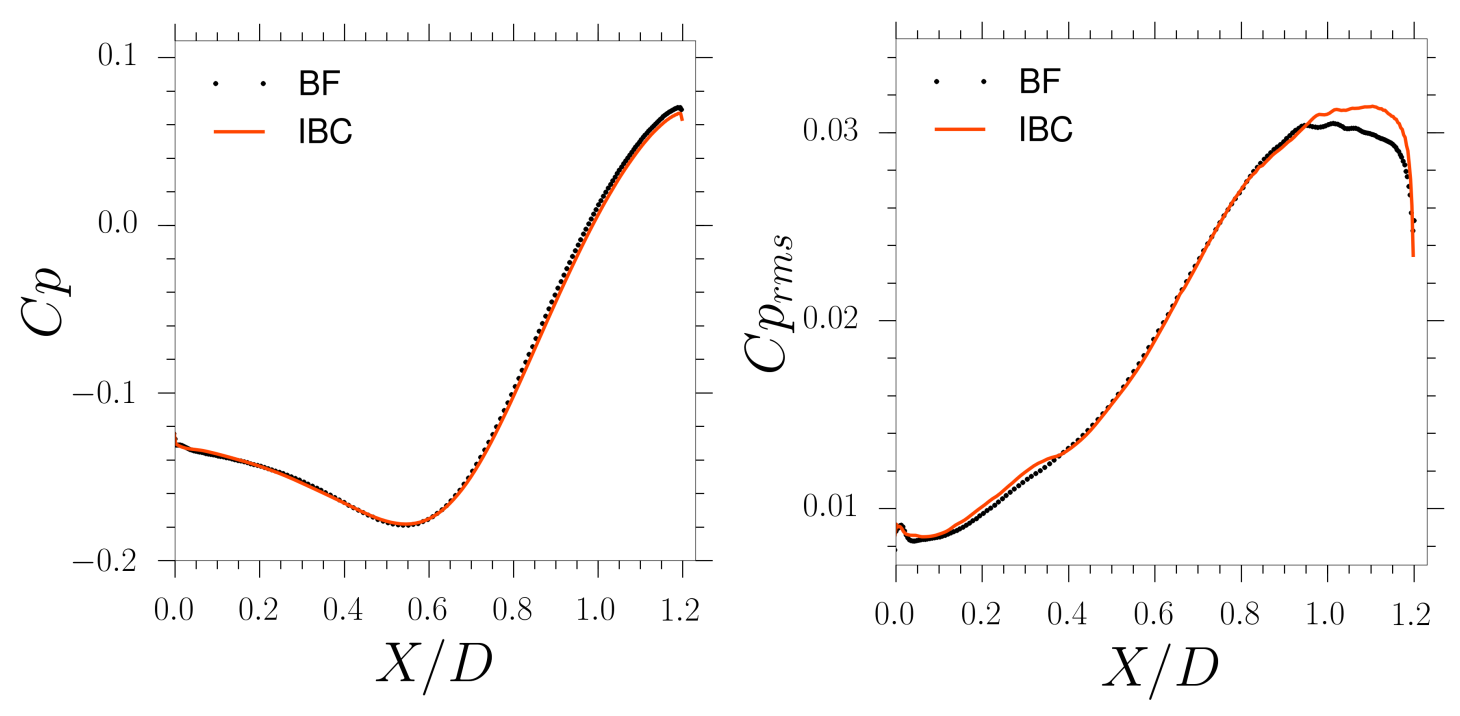

Fig. 6 Streamwise evolution of the mean pressure coefficient (Left) and the rms pressure coefficient (Right) for the GA configuration

\section{B. Global load computation}

The unsteady calculation of the flow field using ZDES coupled with the immersed surface representation allows assessing the fluctuations of the pressure field and its impact on the afterbody load. Figure 8 shows a polar plot of the load coefficient $C f_{z}(t)$ against $C f_{y}(t)$ on the extension for the GA and RA configurations for both body-fitted and IBC simulations. The statistical properties of the dynamic load are assessed using the confidence ellipse of both $F y$ and $F z$ as described by Deck and Nguyen [26] and compared against the validated body-fitted computation. The circular shape of the confidence ellipses reflects the axisymmetry of the configurations and is well-represented for both cases. The ellipse size is also well-reproduced with IBC showing the possibility to quantify the impact of the pressure fluctuations on the space launcher as well as with body-fitted methods.

To go further in the analysis of the global load, figure 9 shows the estimated PSD function of the global load contribution in the $y$ direction for both the generic and the realistic afterbodies. The main frequency peak is located for a Strouhal number of 0.2 , corresponding to the vortex shedding phenomenon at the end of the upstream cylinder. This peak is slightly shifted to a Strouhal number of 0.19 for the realistic afterbody. This variation of the main frequency is well-described by the IBC method. In both configurations, peaks can be found around $S t_{D}=0.4$ which supports the 

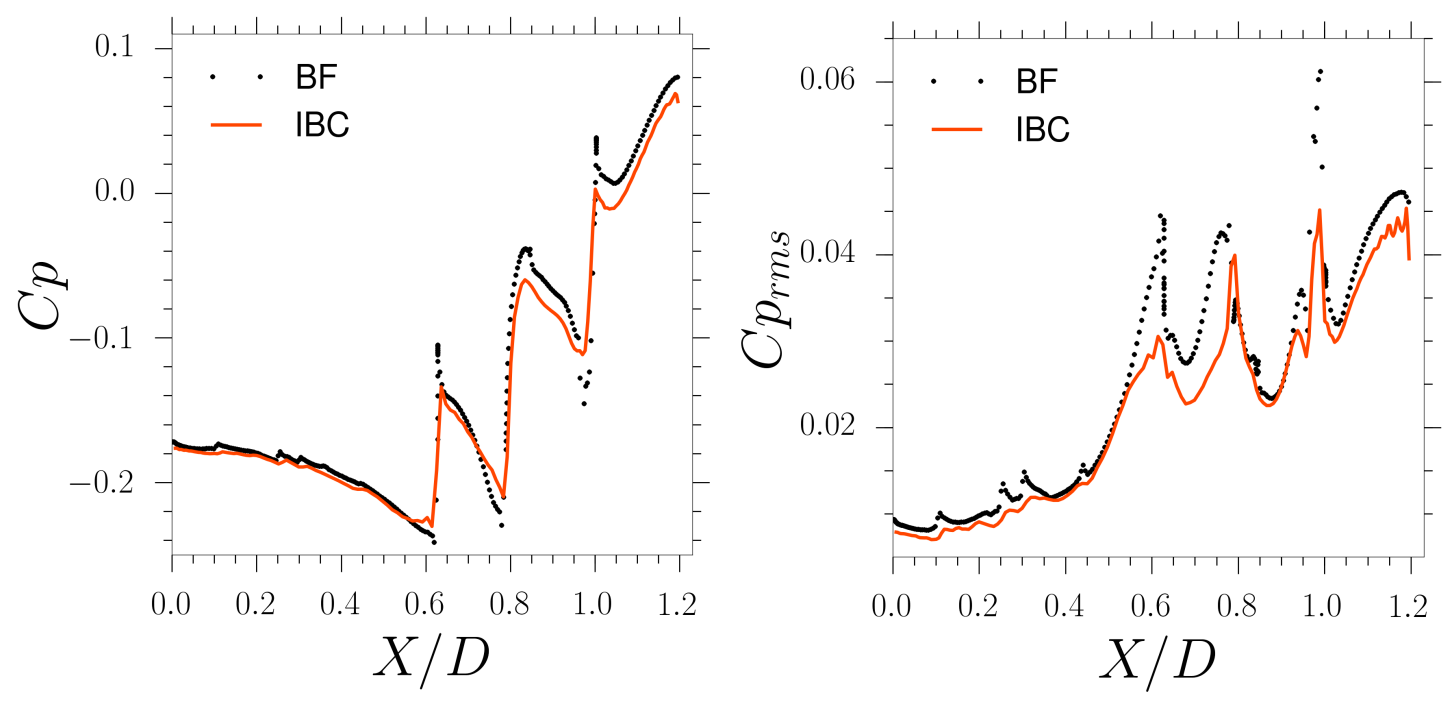

Fig. 7 Streamwise evolution of the mean pressure coefficient (Left) and the rms pressure coefficient (Right) for the RA configuration

capacity of the IBC to describe unsteady load variations.

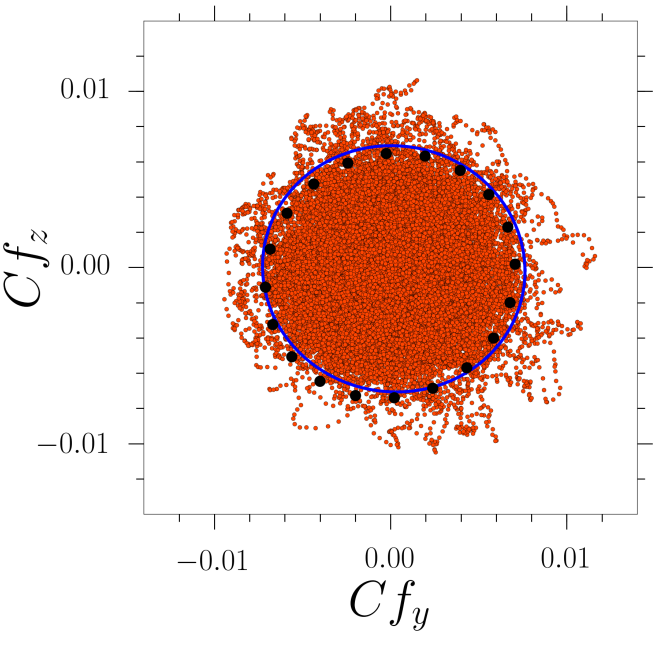

(a) Generic Afterbody (GA)

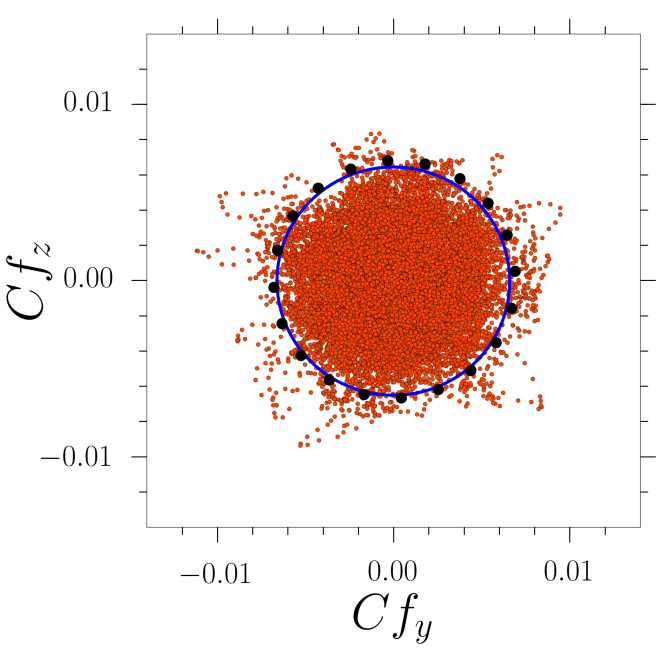

(b) Realistic Afterbody (RA)

Fig. 8 Polar plot of the global load for the IBC simulations $\mathrm{O}$, Confidence ellipse of the IBC simulations Confidence ellipse of the body-fitted simulations

\section{Spatial organisation of the wall pressure fluctuations}

The organisation of the spectral content of the wall pressure field can be obtained using Power Spectral Density (PSD). Such a method is considered as a high level of validation for unsteady simulations as explained by Sagaut and Deck [27]. The IBC capacity to retrieve single-point pressure spectra on its surface has not been assessed yet. The wall extraction presented in Section $\Pi$ was used to sample the wall pressure over a total duration of $200 \mathrm{~ms}$. The PSD was computed along the streamwise direction with a frequency resolution equal to $60 \mathrm{~Hz}$. (see figures 10 and 11 for the GA and RA cases, respectively). For both flows, the three characteristic zones can be distinguished. At the beginning of 


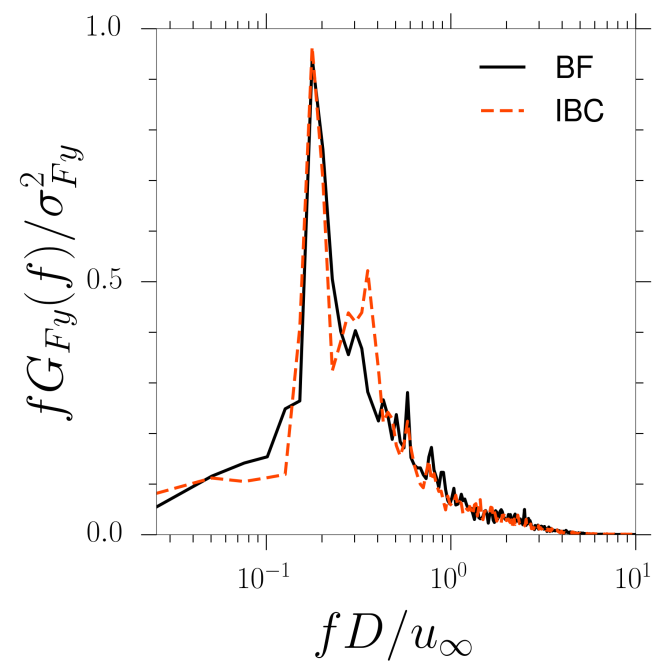

(a) Generic Afterbody (GA)

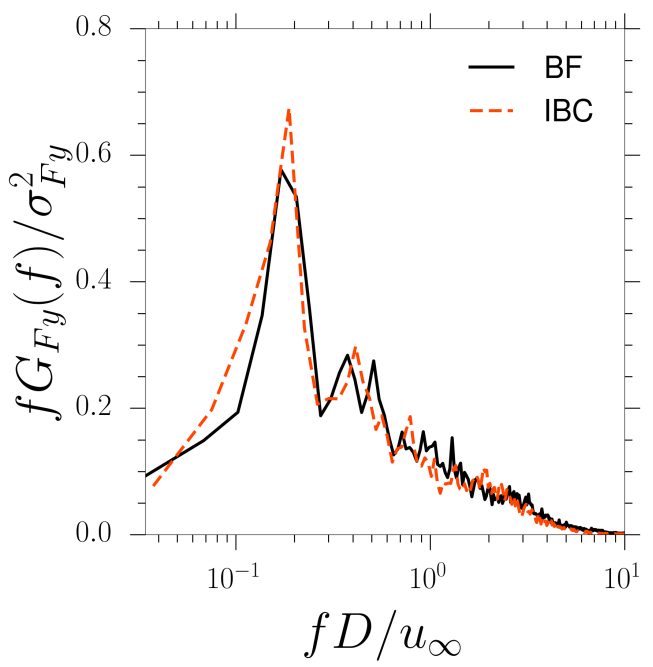

(b) Realistic Afterbody (RA)

Fig. 9 PSD of the side loads for the $y$ component, _- Body-fitted, - - - : IBC

the emergence $(0<x / D<0.2)$ the energy is located at the low frequency below $S t_{d}=0.2$. At the opposite, between $x / D=0.9$ up to 1.2 , the frequency above $S t_{d}=0.4$ is predominant. The main difference between the body-fitted computation and their IBC counterpart is characterized by a shift of the energy to higher frequencies. This difference is consistent with the error of the $C p_{r m s}$ that has been observed in the same area for both configurations (Figs 6 and 7 ). Nonetheless, the main peak of energy located around $x / D=0.55$ is well-reproduced by the IBC simulations for both cases (i.e. GA and RA) at a Strouhal number around 0.2.
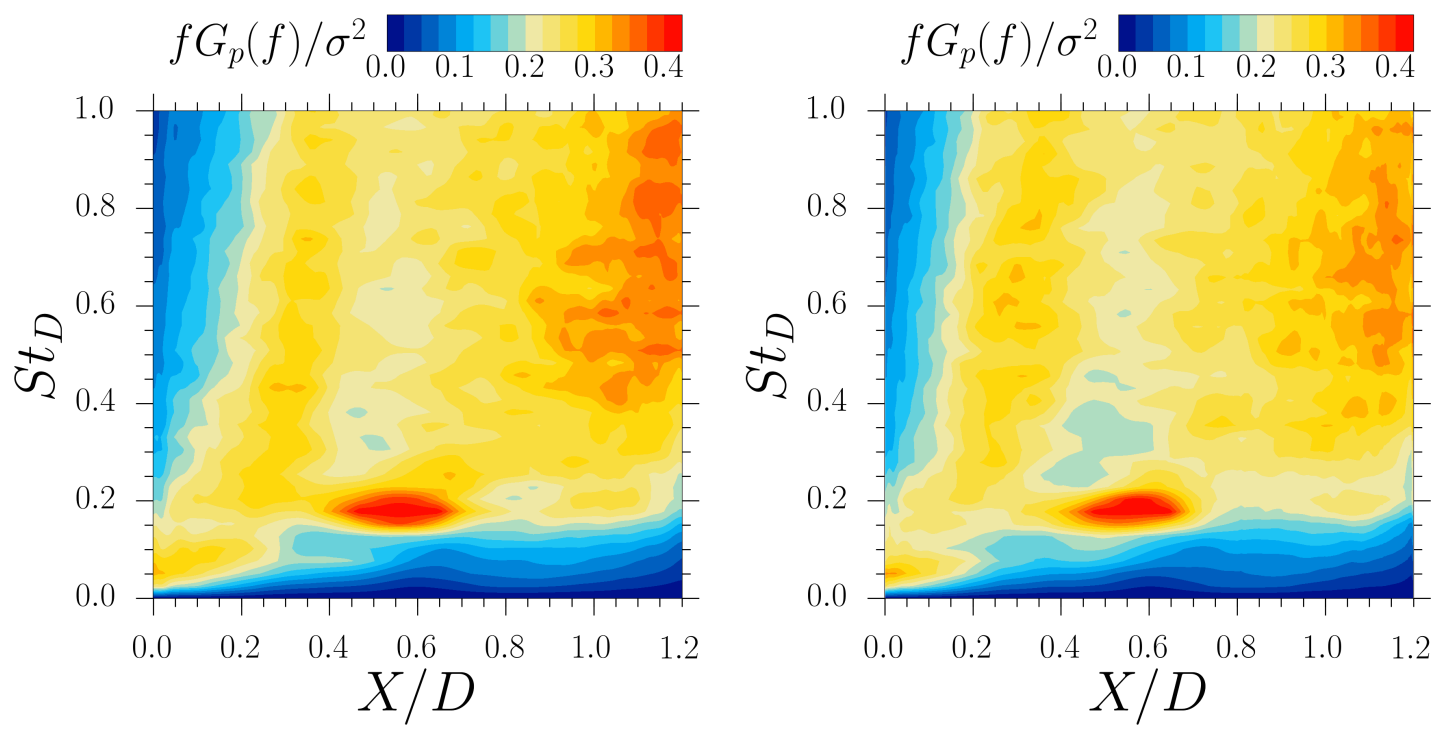

Fig. 10 Maps of the dimensionless power spectral density of the fluctuating pressure for the GA case with Body-Fitted (Left) and Immersed-Boundary (Right) methods.

Two-point spectral analysis is considered for the investigation of the azimuthal coherence of pressure sensors located at angles $\phi_{i}$ distributed around the emergence for every x position. Assuming an homogeneous flow, as proposed by Weiss et al. [13], the complex coherence function can be expressed as :

$$
C(f, r, x, \Delta \phi)=\left(C_{r}+j C_{i}\right)(f, r, x, \Delta \phi)=\frac{S_{12}(f, r, x, \Delta \phi)}{\sqrt{S_{1}(f, r, x, \Delta \phi) S_{2}(f, r, x, \Delta \phi)}}
$$



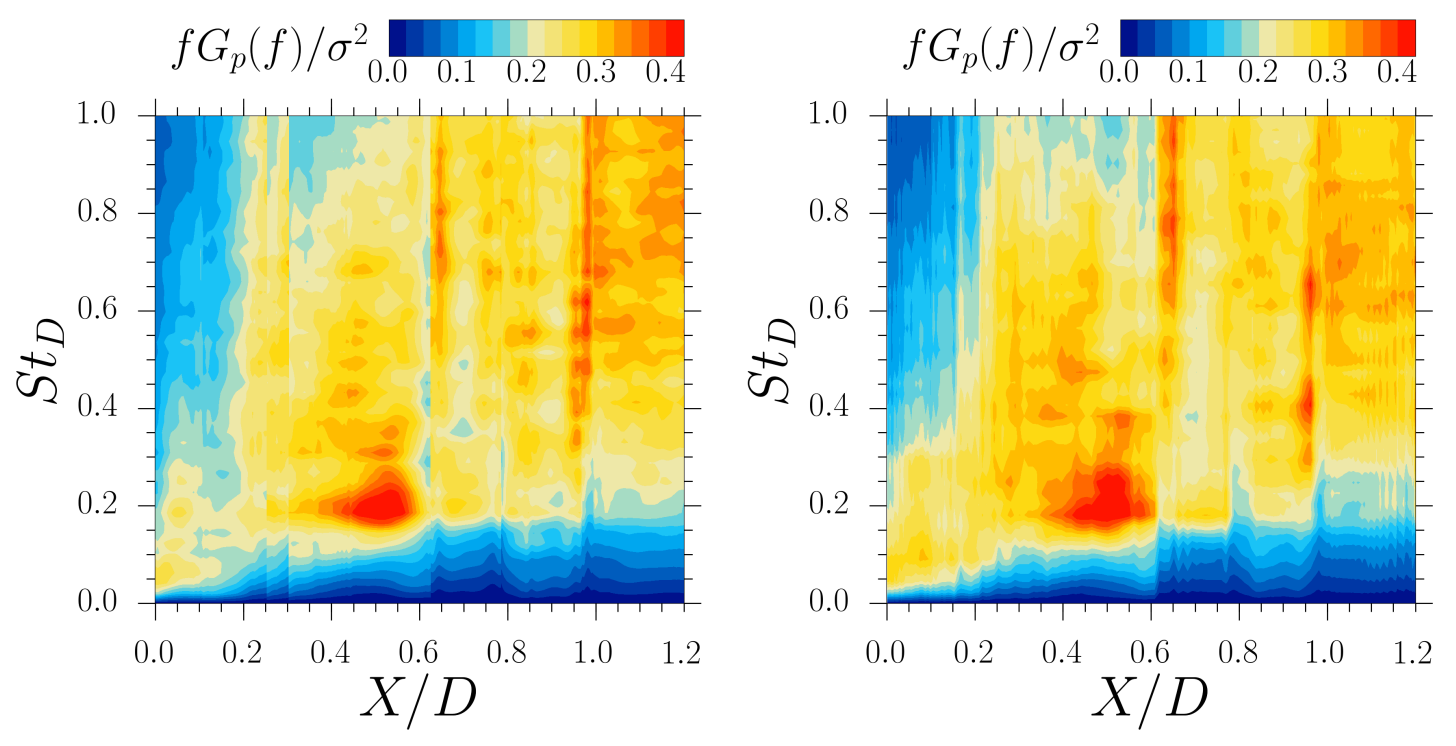

Fig. 11 Maps of the dimensionless power spectral density of the fluctuating pressure for the RA case with Body-Fitted (Left) and Immersed-Boundary (Right) methods.

where $j=\sqrt{-1}$, and $C_{r}$ and $C_{i}$ are the real and imaginary part of the cross-spectral density function $S_{12}$ and $\Delta \phi$ is the angle between two sensors. Assuming an homogeneous propagation of the disturbance and with $C_{i}=0, C_{r}$ is considered as $2 \pi$-periodic and can be expressed as follow:

$$
C_{r}(f, \phi)=\sum_{m=0}^{\infty} C_{r, m}(f) \cos (m \Delta \phi)
$$

Where $C_{r, m}$ is the percentage of the fluctuating energy at the frequency relative to the azimuthal mode $m$. Figures 12, 13 and figures 14, 15 present the comparison of the spectrum for mode 0 and 1, respectively. For both modes the main features are reproduced by the IBC. However, the azimutal coherence is increased for $S t_{D}=0.1$ for both IBC simulations. Such a rise has been observed by Weiss and Deck [2] for the GA case with the presence of four jets near the beginning of the shear layer in order to control the antisymmetric mode $m=1$. The momentum provided by the control device is similar to the action of the source terms for the imposition of the immersed boundary.

\section{Conclusion}

A new methodology to extend the capacity of immersed boundary conditions to reconstruct quantitatively wall quantities has been assessed on two space launcher afterbody configurations using ZDES. An algorithm for the generation of a surface mesh from the knowledge of the IBC has been detailed and tested for both studied configurations. The generation of this surface grid permits to perform the same post-processing as with body-fitted methods without any extra computational cost during the simulations. The results were compared against validated body-fitted simulations using the same schemes and methods. This method is a straightforward way to create integration surfaces for global load computation on immersed boundaries. In particular, the expected variation and magnitude of the global load for the two afterbody configurations of interest are well-reproduced. The surface mesh has also been used for on-the-fly wall extraction in order to process single and two-point spectral analyses. The resulting single point spectral analysis is coherent with the body-fitted simulations which supports the capacity of the IBC to provide an accurate analysis of the flow in the near-wall area. The main features of the two first frequency modes obtained with a two-point spectral analysis have been reproduced with IBC. IBC have shown to introduce a low frequency consistent with the addition of momentum of the source terms used for the immersed boundary imposition. Finally, IBC with adapted post-processing methods constitute a real alternative to body-fitted method for the development of new aerodynamic vehicles. 

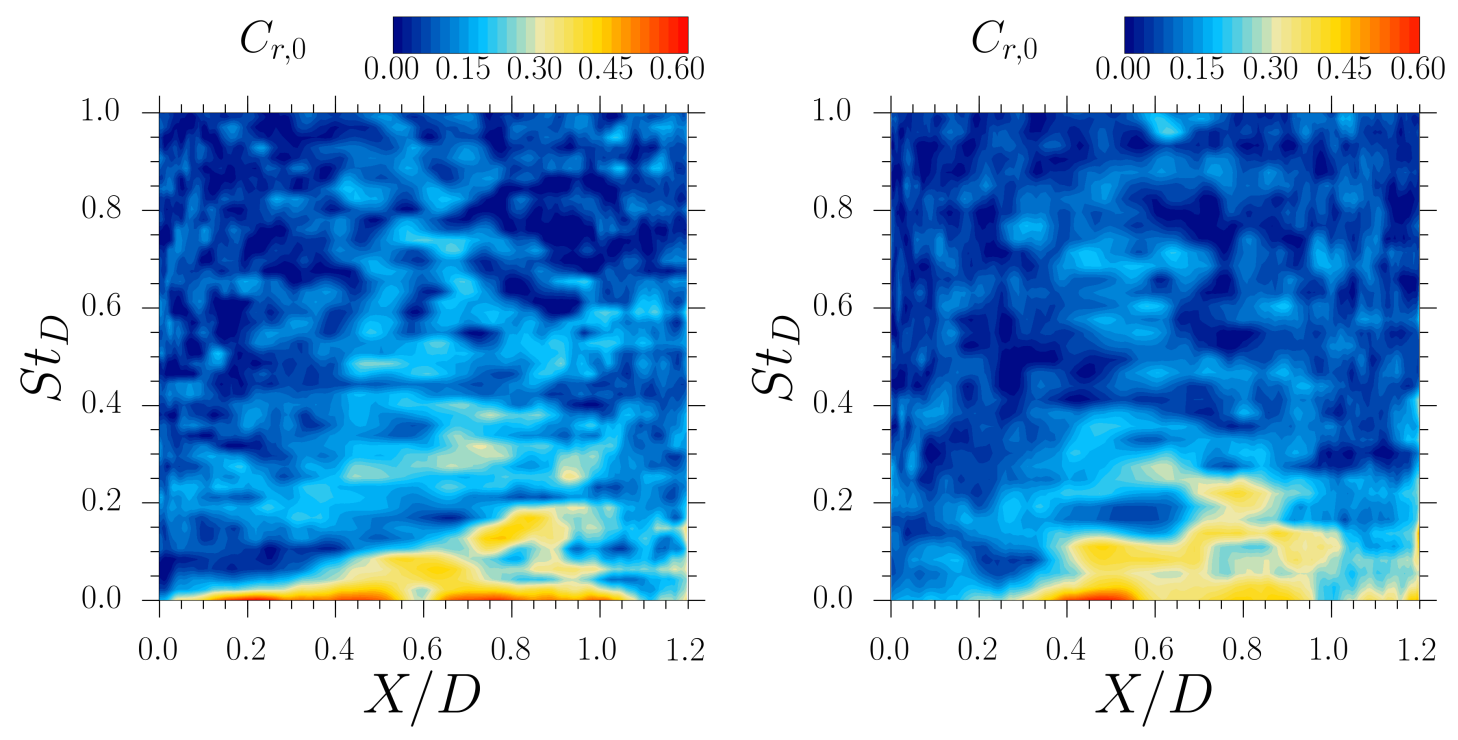

Fig. 12 Maps of the azimuthal pressure mode $C_{r, 0}$ for the GA configuration with Body-Fitted (Left) and Immersed-Boundary (Right) methods.
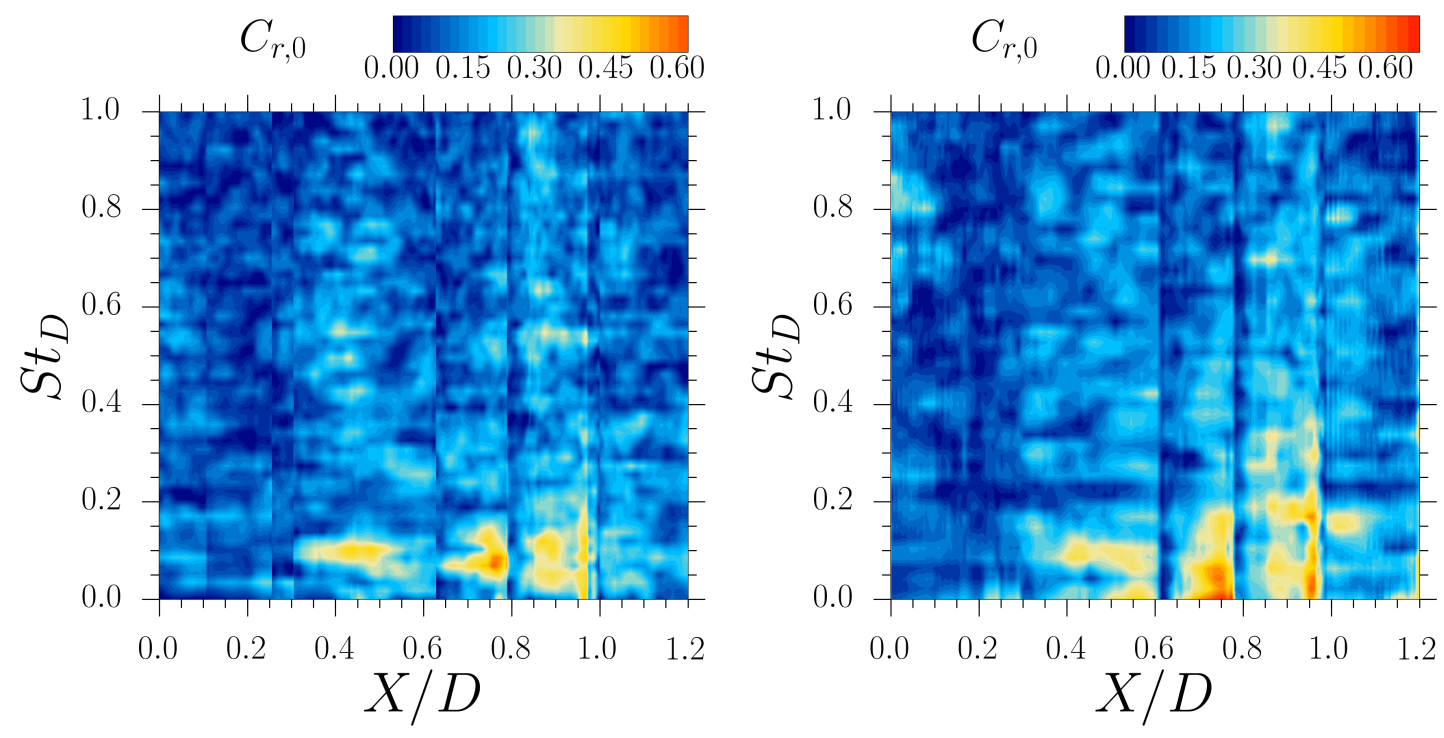

Fig. 13 Maps of the azimuthal pressure mode $C_{r, 0}$ for the RA configuration with Body-Fitted (Left) and Immersed-Boundary (Right) methods.

\section{Appendix}

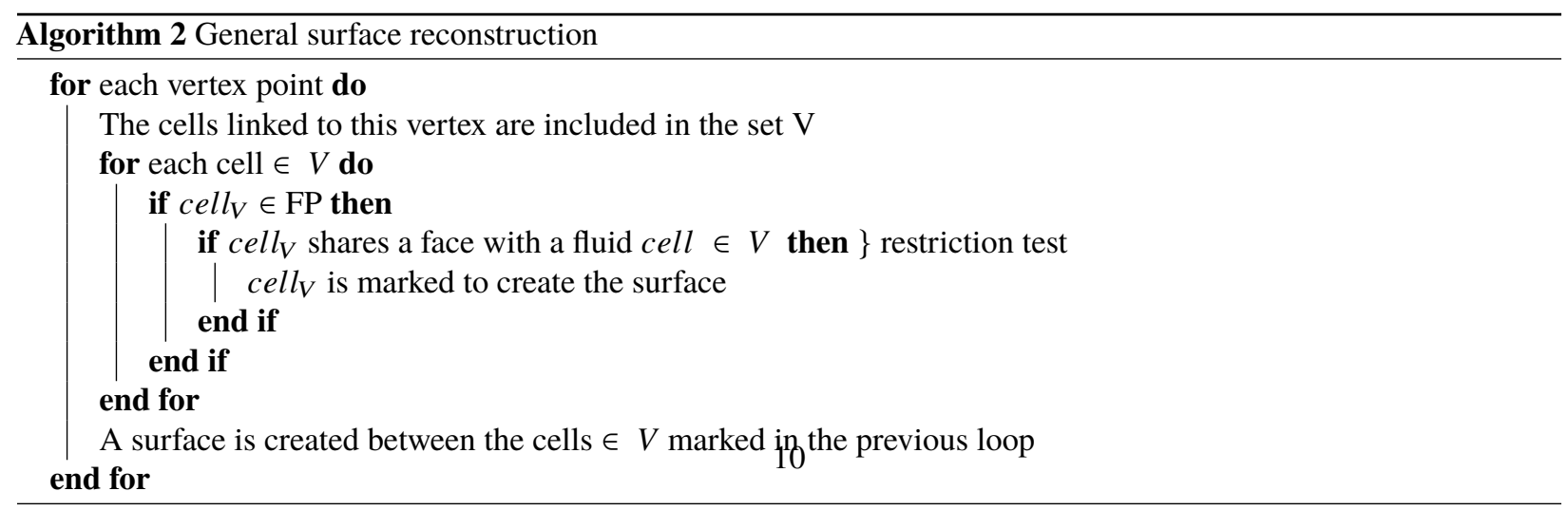



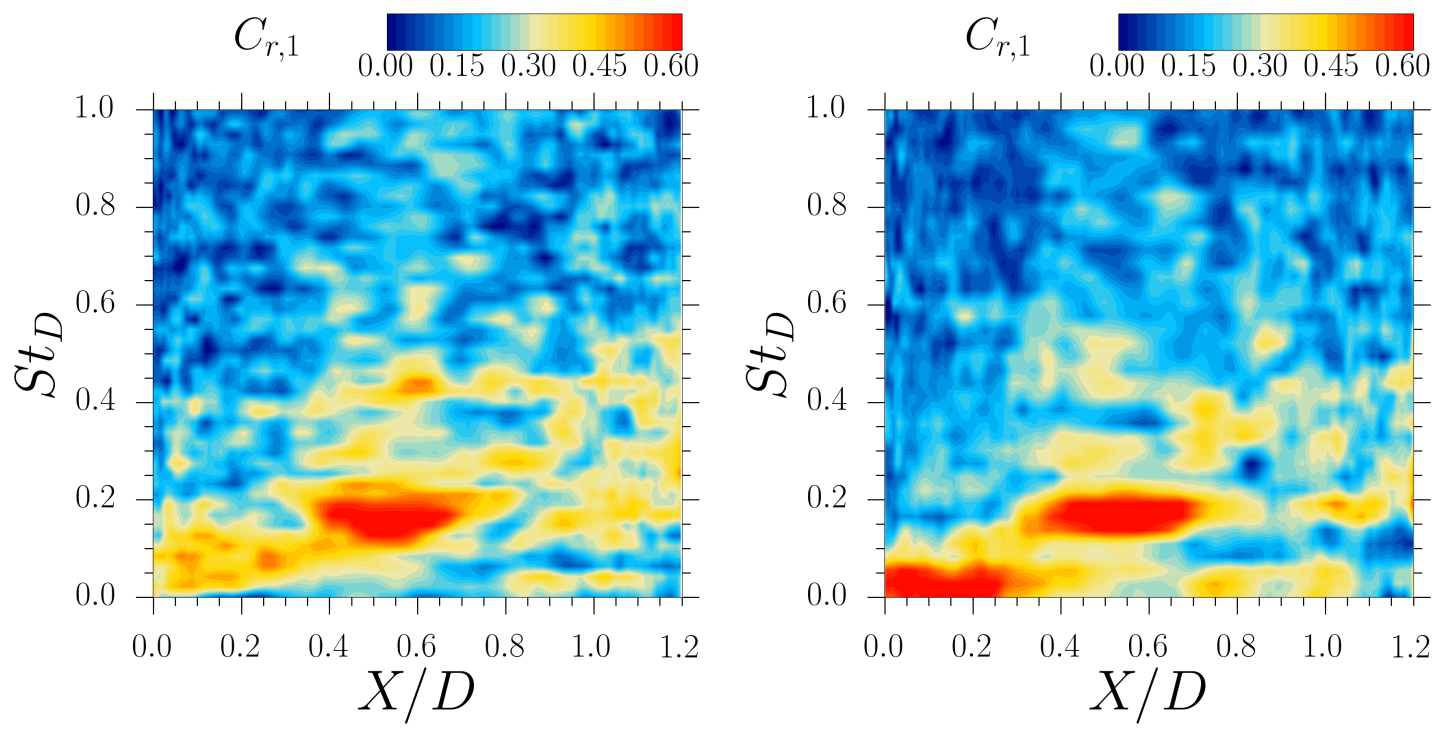

Fig. 14 Maps of the azimuthal pressure mode $C_{r, 1}$ for the GA configuration with Body-Fitted (Left) and Immersed-Boundary (Right) methods.
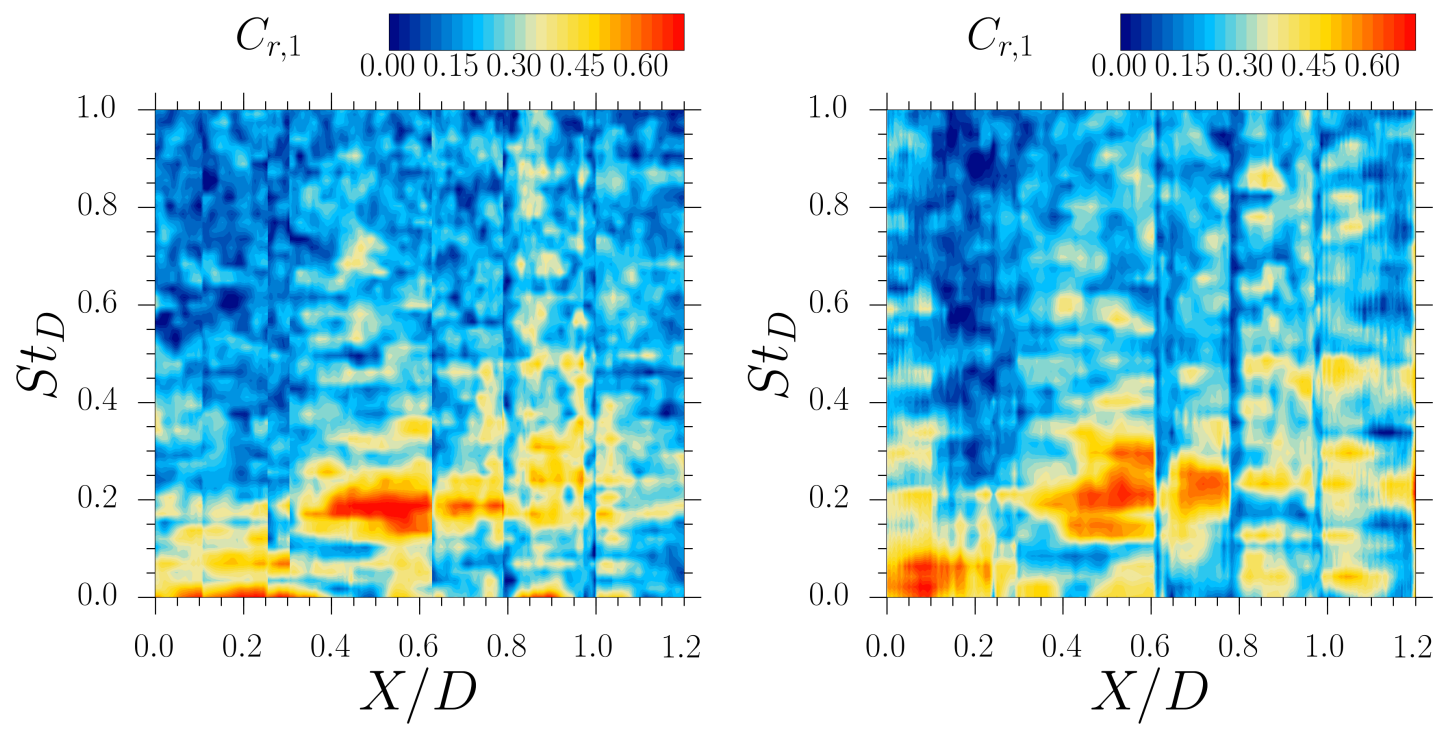

Fig. 15 Maps of the azimuthal pressure mode $C_{r, 1}$ for the RA configuration with Body-Fitted (Left) and Immersed-Boundary (Right) methods.

\section{References}

[1] Deck, S., and Thorigny, P., "Unsteadiness of an axisymmetric separating-reataching flow: Numerical investigation," Physics of Fluids, Vol. 19, No. 065103, 2007, pp. 1-20.

[2] Weiss, P.-É., and Deck, S., "Control of the antisymmetric mode $(\mathrm{m}=1)$ for high Reynolds axisymmetric turbulent separating/reattaching flows," Physics of Fluids, Vol. 23, 2011, p. 095102.

[3] Meliga, P., and Reijasse, P., "Unsteady transonic flow behind an axisymmetric afterbody with two boosters," Proceeding of the 25th AIAA Applied Aerodynamics Conference Miami, 2007, pp. 1-8.

[4] Mochel, L., Weiss, P.-É., and Deck, S., "On the use of Zonal Immersed Boundary Conditions on a high Reynolds number afterbody flow with a serrated skirt using ZDES," 52nd Aerospace Sciences Meeting, American Institute of Aeronautics and Astronautics, 2014. doi:10.2514/6.2014-0046. 
[5] Weiss, P.-É., and Deck, S., "On the coupling of a zonal body-fitted/immersed boundary method with ZDES: Application to the interactions on a realistic space launcher afterbody flow," Computers \& Fluids, 2017, pp. 1-15. doi:10.1016/j.compfluid.2017. 06.015 .

[6] Piquet, A., Roussel, O., and Hadjadj, A., "A comparative study of Brinkman penalization and direct-forcing immersed boundary methods for compressible viscous flows," Computers \& Fluids, Vol. 136, 2016, pp. 272-284.

[7] Brehm, C., Hader, C., and Fasel, H. F., "A locally stabilized immersed boundary method for the compressible Navier-Stokes equations," Journal of Computational Physics, Vol. 295, 2015, pp. 474-504.

[8] Capizzano, F., "Coupling a Wall Diffusion Model with an Immersed Boundary Technique,” AIAA Journal, Vol. 54, No. 2, 2016, pp. 728-734.

[9] Tamaki, Y., and Imamura, T., "Turbulent Flow Simulations of the Common Research Model Using Immersed Boundary Method,” AIAA Journal, 2018, pp. 1-12. doi:10.2514/1.j056654.

[10] Mizuno, Y., Takahashi, S., Nonomura, T., Nagata, T., and Fukuda, K., "A Simple Immersed Boundary Method for Compressible Flow Simulation around a Stationary and Moving Sphere," Mathematical Problems in Engineering, Vol. 2015, 2015 , pp. 1-17.

[11] Wang, K., Rallu, A., Gerbeau, J.-F., and Farhat, C., "Algorithms for interface treatment and load computation in embedded boundary methods for fluid and fluid-structure interaction problems," International Journal for Numerical Methods in Fluids, Vol. 67, 2011, pp. 1175-1206.

[12] Capizzano, F., "Automatic generation of locally refined Cartesian meshes: Data management and algorithms," International Journal for Numerical Methods in Engineering, Vol. 113, No. 5, 2017, pp. 789-813. doi:10.1002/nme.5636.

[13] Weiss, P.-É., Deck, S., Robinet, J.-C., and Sagaut, P., "On the dynamics pf axisymmetric turbulent separating/reattaching flows," Physics of Fluids, Vol. 21, No. 075103, 2009, pp. 1-8.

[14] Weiss, P.-É., and Deck, S., "Zonal Detached Eddy Simulation of the flow dynamics on an Ariane 5-type afterbody," 4th Eur. Conf. for Aerospace Sciences, St-Petersburg, Russia (2011), 2011.

[15] Liou, M.-S., “A Sequel to AUSM: AUSM+,” Journal of Computational Physics, Vol. 129, 1996, p. $364-382$.

[16] Péchier, M., "Prévision numérique de l'effet Magnus pour des configurations de munitions," Ph.D. thesis, Université de Poitiers, 1999.

[17] Dandois, J., Garnier, E., and Sagaut, P., "Unsteady Simulation of Synthetic Jet in a Crossflow,” AIAA Journal, Vol. 44, No. 2, 2006, pp. 225-238. doi:10.2514/1.13462.

[18] Deck, S., "Numerical Simulation of Transonic Buffet over a Supercritical Airfoil," AIAA Journal, Vol. 43, No. 7, 2005 , pp. 1556-1566. doi:10.2514/1.9885.

[19] Deck, S., "Simulation numérique des charges latérales instationnaires sur des configurations de lanceur," Ph.D. thesis, Université d'Orléans, 2002.

[20] Deck, S., Garnier, E., and Guillen, P., “Turbulence modelling applied to space launcher configurations," Journal of Turbulence, Vol. 3, 2002, p. N57. doi:10.1088/1468-5248/3/1/057.

[21] Deck, S., "Zonal-Detached-Eddy Simulation of the flow around a high-lift configuration,” AIAA Journal, Vol. 43, No. 11, 2005, pp. 2372-2384.

[22] Deck, S., "Recent improvements in the Zonal Detached Eddy Simulation (ZDES) formulation," Theoretical and Computational Fluid Dynamics, Vol. 26, No. 6, 2012, pp. 523-550.

[23] Simon, F., Deck, S., Guillen, P., Sagaut, P., and Merlen, A., "Numerical simulation of the compressible mixing layer past an axisymmetric trailing edge,” Journal of Fluid Mechanics, Vol. 591, 2007. doi:10.1017/s0022112007008129.

[24] Mohd-Yusof, J., "Development of immersed boundary methods for complex geometries," Annual Research Briefs, edited by C. for Turbulence Research, 1998, pp. 325-336.

[25] Mochel, L., Weiss, P.-É., and Deck, S., “Zonal Immersed Boundary Conditions: Application to a High-Reynolds-Number Afterbody Flow," AIAA Journal, Vol. 52, No. 12, 2014, p. 2782-2794. 
[26] Deck, S., and Nguyen, A. T., "Unsteady Side Loads in a Thrust-Optimized contour nozzle at hysteresis regime," AIAA Journal, Vol. 42, 2004, pp. 1878-1888.

[27] Sagaut, P., and Deck, S., "Large eddy simulation for aerodynamics: status and perspectives," Philosophical Transactions of the Royal Society A: Mathematical, Physical and Engineering Sciences, Vol. 367, No. 1899, 2009, pp. 2849-2860. doi:10.1098/rsta.2008.0269. 